 \\ TATRA

\section{THE ASYMPTOTIC BEHAVIOUR OF $q$-DIFFERENCE EQUATIONS WITH MULTIPLE DELAYS}

\author{
JAN ČERMÁK
}

\begin{abstract}
We investigate asymptotic properties of solutions of a class of linear $q$-difference equations. We relate their behaviour to the asymptotics of some $q$-exponential functions and mention possible connections with the corresponding continuous case.
\end{abstract}

\section{Introduction and preliminaries}

The discretization of ordinary differential equations is an important process associated with essential problems such as discussions on numerical solutions of these equations or the study of corresponding discrete models. Although the standard way of the discretization utilizes grid points based on an arithmetic progression, a lot of attention has been paid also to q-discretizations related to geometric progressions. This approach forms the background for the theory of q-difference equations which are studied in the frame of quantum calculus. This type of calculus has a long history and the survey of its basic notions, results and methods is summarized, e.g., in [14]. Apart from the traditionality of quantum calculus, many interesting questions and problems either remain open or were answered only recently. Among those related to the discussions on $q$-analogy of some topics in differential equations we can mention, e.g., papers [3] or [19].

The fundamental set forming the base for our next investigations is the geometric progression

$$
q^{\mathbb{Z}}:=\left\{q^{n}, n \in \mathbb{Z}\right\}, \quad \text { or more generally, } \quad \overline{q^{\mathbb{Z}}}:=q^{\mathbb{Z}} \cup\{0\},
$$

2000 Mathematics Subject Classification: 39A12, 39A13, 39B22.

Keywords: $q$-difference equation, $q$-exponential function, asymptotic behaviour.

The research was supported by the grant \# 201/08/0469 of the Czech Grant Agency and by the research plan MSM 0021630518 "Simulation modelling of mechatronic systems" of the Ministry of Education, Youth and Sports of the Czech Republic. 


\section{JAN ČERMÁK}

where $q>1$ is a real number. If $t \in \overline{q^{\mathbb{Z}}}$ and $y: \overline{q^{\mathbb{Z}}} \rightarrow \mathbb{R}$ is a function, then we define the $q$-derivative (or the Jackson derivative) of $y$ at $t$ by

$$
\Delta_{q} y(t):= \begin{cases}\frac{y(q t)-y(t)}{(q-1) t} & \text { if } \quad t \in q^{\mathbb{Z}}, \\ \lim _{s \rightarrow 0} \frac{y(s)-y(0)}{s} & \text { if } \quad t=0\end{cases}
$$

provided the limit exists. By a $q$-difference equation we understand an equation that contains $q$-derivatives of an unknown function defined on $\overline{q^{\mathbb{Z}}}$ (or its subsets).

Similarly we can recall the notion of $q$-integral. If $a, b \in \overline{q^{\mathbb{Z}}}, a<b$, then we define the $q$-integral (or the Jackson integral) by

$$
\int_{a}^{b} f(t) \Delta_{q} t:=\sum_{t \in[a, b) \cap \overline{q^{\mathbb{Z}}}}(q-1) \mathrm{t} f(t) .
$$

Letting $b \rightarrow \infty$ we can arrive at the definition of the corresponding improper integral.

The set $\overline{q^{\mathbb{Z}}}$ is a typical example of a time scale (a nonempty closed subset of real numbers). The previous definitions (as well as many other notions and properties) follow from the general theory of calculus on time scales as particular cases (see [1 and 2]). To illustrate this we recall here the introduction of the $q$-exponential function which is of great importance in our next investigations.

The $q$-exponential function is usually defined in quantum calculus as the $q$ -analogy of the classical exponential function. However, it may not be quite obvious what this $q$-analogy actually means (for two types of $q$-exponential functions see, e.g., [14]). The key property of such a function $y$ that will be required in the next sections is the relation

$$
\Delta_{q} y(t)=y(t), \quad t \in \overline{q^{\mathbb{Z}}} .
$$

If we add the initial condition

$$
y(0)=1 \text {, }
$$

we arrive at the initial value problem (1.1), (1.2). It follows from the time scale theory that this problem has the unique solution $([1])$. We denote it by $\exp _{q}(t)$ and called it the $q$-exponential function. The form of $\exp _{q}(t)$ can be given via the cylinder transformation method. However, we prefer here to find this solution by means of the theory of functional equations in a single variable. We rewrite the equation (1.1) as

$$
y\left(q^{-1} t\right)=\frac{y(t)}{1+\left(1-q^{-1}\right) t}
$$

and consider it as a functional equation with a non-negative continuous time. Then applying Theorem 3.1.13 of [15] we can confirm the existence of the unique 
solution of (1.1), (1.2) and, moreover, find its explicit form

$$
\exp _{q}(t)=\left(1+(q-1) q^{-1} t\right)_{q^{-1}}^{\infty}
$$

where $(1+x)_{q^{-1}}^{\infty}:=\prod_{i=0}^{\infty}\left(1+x q^{-i}\right)$ is the $q^{-1}$-Pochhammer symbol for shifting factorials known from quantum calculus. Note that there are some equivalent expressions for $\exp _{q}(t)$ (see [14]), but in the sequel we utilize the form (1.3).

Now we outline the subject matter of this contribution. We shall be interested in asymptotic investigations of the $q$-difference equation

$$
\Delta_{q} y(t)=\sum_{j=0}^{m} a_{j} t^{\alpha_{j}} y\left(\frac{t}{q^{\beta_{j}}}\right), \quad t \in q^{\mathbb{Z}},
$$

where $a_{j} \neq 0$ and $\alpha_{j}$ are real scalars, $\beta_{j}(j=0, \ldots, m)$ are integers with $0=$ $\beta_{0}<\beta_{1}<\cdots<\beta_{m}$, and $q>1$. This equation can be taken for $q$-analogy of the delay differential equation

$$
y^{\prime}(t)=\sum_{j=0}^{m} a_{j} t^{\alpha_{j}} y\left(\lambda_{j} t\right), \quad t>0,
$$

where $a_{j}, \alpha_{j}, \lambda_{j}$ are real scalars such that $a_{j} \neq 0(j=0, \ldots, m), \lambda_{0}=1$ and $0<\lambda_{j}<1(j=1, \ldots, m)$, which appears along with its modifications in many interesting applications (see, e.g., [16] or [18]). The qualitative investigation of some particular and modified forms of the equation (1.5) has been started in [9, 12, 13, 17, 20] or [4]; other results related directly to the equation (1.5) with polynomial coefficients can be found in [7, 8, and [6].

The equation (1.4) is a $q$-difference equation with multiple delays. The qualitative investigation of delay difference equations is, in general, much less developed than the corresponding theory for delay differential equations. Moreover, it is focused almost entirely in the study of "standard" delay difference equations, i.e., those defined on the time scale with a constant stepsize (see, e.g., [10] and [1] ). Our aim is to extend these investigations also to quantum calculus and present not only some qualitative results on the equation (1.4), but also discuss comparisons with the continuous (differential) case.

\section{The $q$-exponential behaviour of solutions}

The aim of this section is to formulate conditions under which the equation (1.4) admits solutions asymptotically comparable to solutions of a nondelayed $q$-difference equation

$$
\Delta_{q} y(t)=a_{0} t^{\alpha_{0}} y(t) .
$$




\section{JAN ČERMÁK}

First we state the explicit form of a solution of (2.1). Repeating the procedure outlined in Section 1 and assuming that $\alpha_{0}>-1$ we can check that the function

$$
\exp _{q}^{a_{0}, \alpha_{0}}(t):=\left(1+a_{0}(q-1) q^{-\left(\alpha_{0}+1\right)} t^{\alpha_{0}+1}\right)_{q^{-\left(\alpha_{0}+1\right)}}^{\infty}, \quad t \in \overline{q^{\mathbb{Z}}}
$$

defines a solution of (2.1) such that $\exp _{q}^{a_{0}, \alpha_{0}}(0)=1$. Notice that $\exp _{q}^{a_{0}, 0}(t)=$ $\exp _{q}\left(a_{0} t\right)$. If $\alpha_{0} \leq-1$, then the infinite product involved in (2.2) diverges. In such a case we consider $t_{0}=q^{n}$ for a given $n \in \mathbb{Z}$ and introduce this $q$-exponential function by use of the corresponding finite product, namely

$$
\exp _{q}^{a_{0}, \alpha_{0}}(t):=\prod_{s \in\left[t_{0}, t\right) \cap q^{\mathbb{Z}}}\left(1+a_{0}(q-1) s^{\alpha_{0}+1}\right), \quad t \in q^{\mathbb{Z}}, \quad t>t_{0} .
$$

The initial condition now becomes $\exp _{q}^{a_{0}, \alpha_{0}}\left(t_{0}\right)=1$. Of course, this introduction of $\exp _{q}^{a_{0}, \alpha_{0}}$ is possible for any real $\alpha$ and, in our next ideas, it is not necessary to discriminate between (2.2) and (2.3).

The important requirement we impose on this $q$-exponential function is the property

which is equivalent to

$$
\exp _{q}^{a_{0}, \alpha_{0}}(t) \neq 0, \quad t \in q^{\mathbb{Z}}, \quad t \geq t_{0}
$$

$$
1+a_{0}(q-1) t^{\alpha_{0}+1} \neq 0, \quad t \in q^{\mathbb{Z}}, \quad t \geq t_{0} .
$$

Now we are in a position to present the statement relating the asymptotics of (1.4) to the asymptotics of (2.1).

TheOREM 2.1. Consider the equation (1.4), where $a_{j} \neq 0$ and $\alpha_{j}$ are real scalars $(j=0, \ldots, m), 0=\beta_{0}<\beta_{1}<\cdots<\beta_{m}$ are integers and $1+\alpha_{j}<\left(1+\alpha_{0}\right)\left(1+\beta_{j}\right)$ $(j=1, \ldots, m)$. Further, let (2.5) hold. Then for any solution $y$ of (1.4) there exists a constant $L$ such that

$$
\lim _{t \rightarrow \infty} \frac{y(t)}{\exp _{q}^{a_{0}, \alpha_{0}}(t)}=L .
$$

P r o of. Let $y$ be a solution of (1.4). We introduce the change of the dependent variable

Then (1.4) becomes

$$
z(t)=\frac{y(t)}{\exp _{q}^{a_{0}, \alpha_{0}}(t)} .
$$

$$
\Delta_{q} z(t)=\sum_{j=1}^{m} a_{j} t^{\alpha_{j}} \frac{\exp _{q}^{a_{0}, \alpha_{0}}\left(\frac{t}{q^{\beta_{j}}}\right)}{\exp _{q}^{a_{0}, \alpha_{0}}(q t)} z\left(\frac{t}{q^{\beta_{j}}}\right), \quad t \in q^{\mathbb{Z}},
$$

where we have used the $q$-product rule property

$$
\Delta_{q}(f(t) g(t))=\Delta_{q} f(t) g(t)+f(q t) \Delta_{q} g(t) .
$$

For a given $n \in \mathbb{Z}$ now we set $t_{k}:=q^{n+k}$ and $S_{k}:=\sup \left\{\left|z\left(t_{k} q^{-p}\right)\right|, p=\right.$ $\left.0,1, \ldots, k+\beta_{m}\right\}, k=0,1, \ldots$ 
Using (2.7) we can write

$$
z\left(t_{k+1}\right)=z\left(t_{k}\right)+(q-1) \sum_{j=1}^{m} a_{j} t_{k}^{\alpha_{j}+1} \frac{\exp _{q}^{a_{0}, \alpha_{0}}\left(\frac{t_{k}}{q^{\beta_{j}}}\right)}{\exp _{q}^{a_{0}, \alpha_{0}}\left(t_{k+1}\right)} z\left(\frac{t_{k}}{q^{\beta_{j}}}\right),
$$

i.e.,

$$
\left|z\left(t_{k+1}\right)\right| \leq S_{k}\left(1+(q-1) \sum_{j=1}^{m}\left|a_{j}\right| t_{k}^{\alpha_{j}+1}\left|\frac{\exp _{q}^{a_{0}, \alpha_{0}}\left(\frac{t_{k}}{q^{\beta_{j}}}\right)}{\exp _{q}^{a_{0}, \alpha_{0}}\left(t_{k+1}\right)}\right|\right) .
$$

Now calculate the ratio of corresponding $q$-exponential functions as

$$
\begin{aligned}
\left|\frac{\exp _{q}^{a_{0}, \alpha_{0}}\left(\frac{t_{k}}{q^{\beta_{j}}}\right)}{\exp _{q}^{a_{0}, \alpha_{0}}\left(t_{k+1}\right)}\right| & \leq \prod_{p=0}^{\beta_{j}} \frac{1}{\left|1+a_{0}(q-1) q^{-p\left(\alpha_{0}+1\right)} t_{k}^{\alpha_{0}+1}\right|} \\
& =O\left(q^{-k\left(\beta_{j}+1\right)\left(\alpha_{0}+1\right)}\right) \quad \text { as } \quad k \rightarrow \infty .
\end{aligned}
$$

Since $\left(\beta_{j}+1\right)\left(\alpha_{0}+1\right)>\alpha_{j}+1$, we can deduce that

$$
\left|z\left(t_{k+1}\right)\right| \leq S_{k}\left(1+O\left(q^{-\omega k}\right)\right) \quad \text { as } \quad k \rightarrow \infty,
$$

where $\omega>0$ is a suitable constant. Hence

$$
S_{k+1} \leq S_{k}\left(1+O\left(q^{-\omega k}\right)\right) \quad \text { as } \quad k \rightarrow \infty
$$

and the sequence $\left(S_{k}\right)$ is bounded as $k \rightarrow \infty$. Thus $|z(t)| \leq K$ for a suitable positive real $K$ and all $t \in q^{\mathbb{Z}}$.

To prove the statement it is enough to show that $z$ has a (finite) limit. Let $\varepsilon>0$ be arbitrarily small and let $t_{r}, t_{s} \in q^{\mathbb{Z}}, t_{0}<t_{r}<t_{s}$. Rewrite (2.7) as

$$
z\left(t_{s}\right)-z\left(t_{r}\right)=\int_{t_{r}}^{t_{s}} \sum_{j=1}^{m} a_{j} t^{\alpha_{j}} \frac{\exp _{q}^{a_{0}, \alpha_{0}}\left(\frac{t}{q^{\beta_{j}}}\right)}{\exp _{q}^{a_{0}, \alpha_{0}}(q t)} z\left(\frac{t}{q^{\beta_{j}}}\right) \Delta_{q} t,
$$

i.e.,

$$
\left|z\left(t_{s}\right)-z\left(t_{r}\right)\right| \leq K \int_{t_{r}}^{t_{s}} \sum_{j=1}^{m}\left|a_{j}\right| t^{\alpha_{j}}\left|\frac{\exp _{q}^{a_{0}, \alpha_{0}}\left(\frac{t}{q^{\beta_{j}}}\right)}{\exp _{q}^{a_{0}, \alpha_{0}}(q t)}\right| \Delta_{q} t .
$$

Estimating the corresponding Jackson integral by use of (2.8), we can observe its convergency as $t_{s} \rightarrow \infty$. Hence, considering $t_{r}, t_{s}$ large enough, we can guarantee that

$$
\int_{t_{r}}^{t_{s}} \sum_{j=1}^{m}\left|a_{j}\right| t^{\alpha_{j}}\left|\frac{\exp _{q}^{a_{0}, \alpha_{0}}\left(\frac{t}{q^{\beta_{j}}}\right)}{\exp _{q}^{a_{0}, \alpha_{0}}(q t)}\right| \Delta_{q} t<\frac{\varepsilon}{K}
$$

which implies that $\left|z\left(t_{s}\right)-z\left(t_{r}\right)\right|<\varepsilon$, hence $z$ is tending to a finite limit. 
Corollary 2.2. Consider the equation

$$
\Delta_{q} y(t)=\sum_{j=0}^{m} a_{j} y\left(\frac{t}{q^{j}}\right), \quad t \in q^{\mathbb{Z}},
$$

where $a_{j} \neq 0$ are real scalars. Then for any solution $y$ of (2.9) there exists a constant $L$ such that

$$
\lim _{t \rightarrow \infty} \frac{y(t)}{\exp _{q}\left(a_{0} t\right)}=L
$$

\section{Other types of asymptotics of solutions}

In this section we wish to extend the asymptotic description of solutions of (1.4) also to other choices of entry parameters. In particular, throughout this section we assume that $a_{0}<0$ and either $\alpha_{j}>\alpha_{0}$ or $\alpha_{j} \geq \alpha_{0}, a_{0}+\sum_{j=1}^{m}\left|a_{j}\right| \geq 0$ $(j=1, \ldots, m)$. Another requirement we are going to employ in this section is positivity of corresponding $q$-exponential functions. To guarantee this property we have to modify the relation (2.4) as

$$
\exp _{q}^{a_{0}, \alpha_{0}}(t)>0, \quad t \in q^{\mathbb{Z}}, \quad t \geq t_{0},
$$

i.e.,

$$
1+a_{0}(q-1) t^{\alpha_{0}+1}>0, \quad t \in q^{\mathbb{Z}}, \quad t \geq t_{0} .
$$

Keeping in mind the possible analogy with the continuous case, we consider the auxiliary relation

$$
a_{0} t^{\alpha_{0}} \varphi(t)+\sum_{j=1}^{m}\left|a_{j}\right| t^{\alpha_{j}} \varphi\left(\frac{t}{q^{\beta_{j}}}\right) \leq 0, \quad t \in q^{\mathbb{Z}} .
$$

It is known that functional equations and inequalities of this type are of great importance in qualitative investigations of delay equations. There exist several methods of their solving (methods of invariants, Mellin transform method). Applying these procedures and assuming that the above stated conditions on $a_{j}$ and $\alpha_{j}$ are valid we can find the solution of (3.2) in the form

$$
\varphi(t)=\exp \left\{\frac{\left(\alpha-\alpha_{0}\right)\left(\log ^{2} t+\beta_{1} \log q \log t\right)-2 \log \left(\frac{-a_{0}}{a}\right) \log t}{2 \beta_{1} \log q}\right\},
$$

where $\alpha:=\max \left(\alpha_{1}, \ldots, \alpha_{m}\right)$ and $a:=\sum_{j=1}^{m}\left|a_{j}\right|$.

The significance of the inequality (3.2) in the asymptotic investigation of (1.4) presents the following 
TheOREM 3.1. Consider the equation (1.4), where $a_{j} \neq 0$ and $\alpha_{j}$ are real scalars, $a_{0}<0$ and $0=\beta_{0}<\beta_{1}<\cdots<\beta_{m}$ are integers. Further, let either $\alpha_{j}>\alpha_{0}$ or $\alpha_{j} \geq \alpha_{0}, a_{0}+\sum_{j=1}^{m}\left|a_{j}\right| \geq 0(j=1, \ldots, m)$, let (3.1) hold and let $\varphi$ be given by (3.3). Then

$$
y(t)=O(\varphi(t)) \quad \text { as } \quad t \rightarrow \infty
$$

for any solution $y$ of (1.4).

Proof. Let $y$ be a solution of (1.4) and let $z(t)=y(t) / \varphi(t)$. Then

$$
\Delta_{q} z(t) \varphi(t)+z(q t) \Delta_{q} \varphi(t)=\sum_{j=0}^{m} a_{j} t^{\alpha_{j}} \varphi\left(\frac{t}{q^{\beta_{j}}}\right) z\left(\frac{t}{q^{\beta_{j}}}\right),
$$

hence

$$
\Delta_{q}\left[\frac{z(t) \varphi(t)}{\exp _{q}^{a_{0}, \alpha_{0}}(t)}\right]=\sum_{j=1}^{m} \frac{a_{j} t^{\alpha_{j}} \varphi\left(\frac{t}{q^{\beta_{j}}}\right) z\left(\frac{t}{q^{\beta_{j}}}\right)}{\exp _{q}^{a_{0}, \alpha_{0}}(q t)}
$$

by use of the $q$-quotient rule property

$$
\Delta_{q}\left(\frac{f(t)}{g(t)}\right)=\frac{\Delta_{q} f(t) g(t)-f(t) \Delta_{q} g(t)}{g(t) g(q t)} .
$$

Let the symbols $t_{k}$ and $S_{k}$ have the same meaning as in the proof of Theorem 2.1, i.e., $t_{k}:=q^{n+k}$ and $S_{k}:=\sup \left\{\left|z\left(t_{k} q^{-p}\right)\right|, p=0,1, \ldots, k+\beta_{m}\right\}, k=0,1, \ldots$ for an positive integer $n$. Then

$$
\begin{aligned}
z\left(t_{k+1}\right)= & \frac{\varphi\left(t_{k}\right) \exp _{q}^{a_{0}, \alpha_{0}}\left(t_{k+1}\right)}{\varphi\left(t_{k+1}\right) \exp _{q}^{a_{0}, \alpha_{0}}\left(t_{k}\right)} z\left(t_{k}\right) \\
& +(q-1) t_{k} \frac{\exp _{q}^{a_{0}, \alpha_{0}}\left(t_{k+1}\right)}{\varphi\left(t_{k+1}\right)} \sum_{j=1}^{m} \frac{a_{j} t_{k}^{\alpha_{j}} \varphi\left(\frac{t_{k}}{q^{\beta_{j}}}\right) z\left(\frac{t_{k}}{q^{\beta_{j}}}\right)}{\exp _{q}^{a_{0}, \alpha_{0}}\left(t_{k+1}\right)}
\end{aligned}
$$

and taking absolute values we get

$$
\left|z\left(t_{k+1}\right)\right| \leq S_{k} \frac{\varphi\left(t_{k}\right) \exp _{q}^{a_{0}, \alpha_{0}}\left(t_{k+1}\right)}{\varphi\left(t_{k+1}\right) \exp _{q}^{a_{0}, \alpha_{0}}\left(t_{k}\right)}+S_{k}(q-1) t_{k} \sum_{j=1}^{m} \frac{\left|a_{j}\right| t_{k}^{\alpha_{j}} \varphi\left(\frac{t_{k}}{q^{\beta_{j}}}\right)}{\varphi\left(t_{k+1}\right)} .
$$

Now we employ the inequality (3.1) and accomplish the direct calculations of the corresponding $q$-exponential functions. Then

$$
(q-1) t_{k} \sum_{j=1}^{m} \frac{\left|a_{j}\right| t_{k}^{\alpha_{j}} \varphi\left(\frac{t_{k}}{q^{\beta_{j}}}\right)}{\varphi\left(t_{k+1}\right)} \leq \frac{-a_{0}(q-1) t_{k}^{\alpha_{0}+1} \varphi\left(t_{k}\right)}{\varphi\left(t_{k+1}\right)}
$$




\section{JAN ČERMÁK}

and substituting into (3.5) we arrive at the estimate

$$
\begin{aligned}
\left|z\left(t_{k+1}\right)\right| & \leq S_{k} \frac{\varphi\left(t_{k}\right)}{\varphi\left(t_{k+1}\right)}\left[a_{0}(q-1) t_{k}^{\alpha_{0}+1}+1-a_{0}(q-1) t_{k}^{\alpha_{0}+1}\right] \\
& =S_{k} \frac{\varphi\left(t_{k}\right)}{\varphi\left(t_{k+1}\right)}
\end{aligned}
$$

by use of the positivity of corresponding $q$-exponential functions. The properties imposed on coefficients $\alpha_{j}$ and $a_{j}$ now imply that $\varphi$ must be nondecreasing, hence $\left|z\left(t_{k+1}\right)\right| \leq S_{k}$ and $S_{k+1} \leq S_{k}, k=1,2, \ldots$ The boundedness of $z$ now yields the asymptotic property (3.4).

Remark 3.2. The conditions of Theorem 3.1 do not involve the case when $\varphi$ is decreasing. We show that the estimate (3.4) actually need not be valid in such a case.

EXAMPLE 3.3. Consider the equation (1.4) with $q=2, m=\beta_{1}=1, a_{0}=-1 / 2$ and $\alpha_{0}=\alpha_{1}=-1$, i.e., we are given by the equation

$$
\Delta_{2} y(t)=-\frac{1}{2 t} y(t)+\frac{a_{1}}{t} y\left(\frac{t}{2}\right), \quad t \in 2^{\mathbb{Z}}
$$

and assume that $a_{1}>0$. Then the inequality (3.2) can be simplified as

$$
-\frac{\varphi(t)}{2}+a_{1} \varphi\left(\frac{t}{2}\right) \leq 0
$$

which is the Schröder inequality. The formula (3.3) yields the function

$$
\varphi(t)=t^{\gamma}, \quad \gamma=\frac{\log a_{1}}{\log 2}+1
$$

satisfying (3.7) in the form of the equality. However, (3.6) admits the solution

$$
y(t)=t^{\delta}, \quad \delta=\frac{\log \left(1+\sqrt{1+16 a_{1}}\right)}{\log 2}-2
$$

and it is easy to check that $\delta \leq \gamma$ if and only if $a_{1} \geq 1 / 2$. In other words, the asymptotic result (3.4) fails provided $a_{1}<1 / 2$ (i.e., in the asymptotic stable case).

\section{Concluding remarks}

In previous sections we described some types of asymptotics of $q$-difference equation (1.4). Its asymptotic behaviour was related (under given conditions on parameters) to the behaviour of a special $q$-exponential function as well as to the behaviour of some other functions (in a particular case also algebraic). 
The natural question concerning discrete analogues of originally continuous models is the problem of possible similarities or discrepancies in the qualitative behaviour of continuous and discretized equations. As we have remarked in the introductory section, basic results on the asymptotics of solutions of the differential equation (1.5) are already known. It is perhaps not surprising that this equation admits the solutions with asymptotics of exponential type (in other words, $q$-exponential function becomes the "classical" exponential function as $q \rightarrow 1$ from the right). However, this type of the behaviour of solutions is restricted on equations (1.5) with a positive coefficient $a_{0}$. We recall that Theorem 2.1 discussing the $q$-exponential behaviour of (1.4) does not require any condition of this type. On the other hand, the condition (2.5) involved in Theorem 2.1 has no analogy in the continuous case.

The second type of asymptotics of (1.4), stated in Section 3, has been formulated (among others) under the condition $a_{0}<0$. Note that the same sign condition as well as the same majorant function $\varphi$ appear also in the asymptotic investigation of (1.5). However, there is no assumption of the type (3.1) in the relevant results on the equation (1.5). The intuitive explanation is clear: the "classical" exponential function is positive and any additional assumptions of this type are useless.

These questions can be generalized also to other discrete settings of the differential equation (1.5). It is just the time scale theory which turns out to be an efficient tool in these investigations. We recall that some of the above sketched discrepancies between (1.4) and (1.5) can be formulated (and partly explained) using this general approach (in particular: assumptions (2.5) and (3.1) are nothing else, but the conditions of regressivity and positive regressivity, guaranteeing that the corresponding exponential function has a nonzero and positive sign, respectively). For some preliminary results of this type see [5].

Of course, our investigations can be extended also in the frame of $q$-difference equations. The possible extensions of our results may concern other choices of entry parameters (in particular, the case when the upper bound function $\varphi$ from (3.4) is decreasing) or the precision of the above formulated asymptotic results.

\section{REFERENCES}

[1] BOHneR, M.-PETERSOn, A.: Dynamic Equations on Time Scales. An Introduction with Applications. Birkhäuser, Basel, 2001.

[2] BOHneR, M.-Peterson, A.: Advances in Dynamic Equations on Time Scales. Birkha̋user, Boston, MA, 2003.

[3] BOHNER, M.-ÜNAL, M.: Kneser's theorem in q-calculus, J. Phys. A 38 (2005), $6729-6739$. 


\section{JAN ČERMÁK}

[4] ČERMÁK, J.: The asymptotic bounds of solutions of linear delay systems, J. Math. Anal. Appl. 225 (1998), 373-388.

[5] ČERMÁK, J.: On the asymptotics of solutions of delay dynamic equations on time scales, Math. Comp. Modelling 46 (2007), 455-458.

[6] ČERMÁK, J.: On the differential equation with power coefficients and proportional delays, Tatra Mt. Math. Publ. 38 (2007), 57-69.

[7] DERFEL, G.: Functional-differential equations with compressed arguments and polynomial coefficients: asymptotics of the solutions, J. Math. Anal. Appl. 193 (1995), 671-679.

[8] DERFEL, G.-VOGL, F.: On the asymptotics of solutions of a class of linear functional-differential equations, European J. Appl. Math. 7 (1996), 511-518.

[9] DIBLÍK, J.: Asymptotic behaviour of solutions of linear differential equations with delay, Ann. Polon. Math. LVIII (1993), 131-137.

[10] ELAYDI, S. N.: An Introduction to Difference Equations. Undergrad. Texts Math., Springer-Verlag, New York, NY, 1995.

[11] GYÖRI, I.-PITUK, M.: Comparison theorems and asymptotic equilibrium for delay differential and difference equations, Dynam. Systems Appl. 5 (1996) 277-302.

[12] ISERLES, A.: On the generalized pantograph functional-differential equation, European J. Appl. Math. 4 (1993), 1-38.

[13] KATO, T.-MCLEOD, J. B.: The functional differential equation $y^{\prime}(x)=a y(\lambda x)+b y(x)$, Bull. Amer. Math. Soc. (N.S.) 77 (1971), 891-937.

[14] KAC, V.-CHEUNG, P.: Quantum Calculus. Universitext, Springer-Verlag, New York, NY, 2002.

[15] KUCZMA, M.-CHOCZEWSKI, B.-GER, R.: Iterative Functional Equations. Encyclopedia Math. Appl., Cambridge University Press, Cambridge, 1990.

[16] LEHNINGER, H.-LIU, Y.: The functional-differential equation $y^{\prime}(t)=A y(t)+B y(q t)+$ $C y^{\prime}(q t)+f(t)$, European J. Appl. Math. 9 (1998), 81-91.

[17] MAKAY, G.-TERJÉKI, J.: On the asymptotic behavior of the pantograph equations, Electron. J. Qual. Theory Differ. Equ. 2 (1998), 1-12 (electronic).

[18] OCKENDON, J. R.-TAYLER A. B.: The dynamics of a current collection system for an electric locomotive, Proc. Roy. Soc. London Ser. A. 322 (1971), 447-468.

[19] NAGATOMO, K.-KOGA, Y.: q-difference analogue of the Euler-Poisson-Darboux equation and its Laplace sequence, Osaka J. Math. 32 (1995), 451-465.

[20] PANDOLFI, L.: Some observations on the asymptotic behaviors of the solutions of the equation $x^{\prime}(t)=A(t) x(\lambda t)+B(t) x(t), \lambda>0$, J. Math. Anal. Appl. 67 (1979), 483-489.

Received October 29, 2008

Institute of Mathematics
FME BUT
Technická 2
CZ-616-69 Brno
CZECH REPUBLIC

E-mail: cermak.j@fme.vutbr.cz 